\title{
Tuberculosis in the Peritoneum: Not Too Rare After All
}

\author{
Michael Karanikas ${ }^{a} \quad K^{2}$ stantinos Porpodis ${ }^{d}$ \\ Paul Zarogoulidis ${ }^{d}$ Alexandros Mitrakas $^{a}$ \\ Panagiotis Touzopoulos ${ }^{a}$ Nikolaos Lyratzopoulos $^{a}$ \\ George Kouklakis $^{b}$ Nikolaos Courcoutsakis ${ }^{c}$ \\ Alexandros Polychronidis ${ }^{a}$
}

\begin{abstract}
${ }^{a}$ 1st University Surgery Department, ${ }^{b}$ Gastrointestinal Endoscopy Unit and ${ }^{\mathrm{C}}$ Radiology Department, University General Hospital of Alexandroupolis,

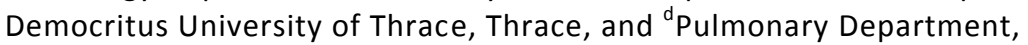
'G. Papanikolaou' General Hospital, Aristotle University of Thessaloniki, Thessaloniki, Greece
\end{abstract}

\section{Key Words}

Abdominal · Tumor · Ascites · Laparoscopy

\begin{abstract}
Peritoneal tuberculosis is rare with increased incidence rates in recent years. The absence of characteristic clinical features of the disease often makes its diagnosis difficult and elusive. We present the case of 61-year-old female with peritoneal tuberculosis. The patient suffered from abdominal pain for a period of 5 months prior to admission. The diagnosis was established on the basis of findings from an abdominal computed tomography scan, a chest radiograph and histopathological analysis of the laparoscopic resection of the two masses. The patient was discharged from hospital receiving a fourfold antituberculous treatment with isoniazid, rifampicin, pyrazinamide and ethambutol. A high index of suspicion and a combination of radiologic, endoscopic, microbiologic and histopathological examination achieves diagnostic accuracy and prevents clinical mismanagement.
\end{abstract}

\section{Introduction}

The rates of tuberculosis (TB) continue to rise throughout the world causing 6\% mortality. TB has become a public health problem worldwide [1]. The abdominal form is the sixth leading cause of extrapulmonary TB, after lymphatic, genitourinary, 
osteoarticular, miliary and meningeal [2]. Abdominal TB can infect any part of the gastrointestinal track including the peritoneum and the pancreatobiliary system with Mycobacterium tuberculosis [1].

Abdominal TB is predominant in individuals $<40$ years of age, with greater frequency in females [3]. Clinical manifestations are nonspecific, frequently mimicking other abdominal diseases such as inflammatory intestinal disease, advanced ovarian cancer, deep mycosis, Yersinia infection and amebomas [3]. The clinical presentation can be acute or chronically intermittent. Most patients (80-95\%) have abdominal pain, $40-90 \%$ fever, $11-20 \%$ diarrhea and constipation, and $40-90 \%$ weight loss, anorexia and malaise [4]. Patients with peritoneal TB manifest a slowly progressive abdominal swelling from ascites and abdominal pain. Adhesions may also cause small intestine obstruction. On physical examination, diffuse abdominal tenderness, doughy abdomen, hepatomegaly and ascites may also be present. The risk factors for developing peritoneal TB are HIV infection, cirrhosis, diabetes, malignancy and receiving continuous ambulatory peritoneal dialysis [5].

Peritoneal TB occurs in three types: (i) the wet type with ascites, (ii) the encysted type with abdominal swelling, and (iii) the fibrotic type with abdominal masses composed of mesenteric and omental thickening. A combination of these types may also occur [4]. During infection, the peritoneum becomes thick, hyperemic and less shiny. Both visceral and parietal peritoneal layers are studded with multiple tuberculous nodules [5]. Ascites manifest secondarily as the proteinaceous fluid exudates from the tubercles. Approximately $90 \%$ of patients with TB peritonitis suffer from ascites [5]. Pathogenesis involves infection by tubercle bacilli (a) following reactivation via hematogenous spread from a primary lung focus, (b) via hematogenous spread from active pulmonary or miliary TB, and (c) through lymph channels from infected nodes [5]. Peritoneal involvement may occur from infected contiguous lymph nodes, intestinal lesions or fallopian tubes in women. Additionally, abdominal lymph nodal and peritoneal TB may develop without gastrointestinal involvement in $30 \%$ of cases [5].

\section{Case Report}

A 61-year-old female was admitted to the surgical department with a 5-month history of abdominal pain. The patient was apyretic and initial laboratory data showed low hemoglobin $(11.3 \mathrm{~g} / \mathrm{dl})$, normal white blood cells $(4,810 / \mu \mathrm{l})$, increased erythrocyte sedimentation rate $(45 \mathrm{~mm})$ and normal blood gas levels. On physical examination, the patient's abdomen was soft, pliable, with normal bowel sounds and without ascites. Chest radiograph did not present any infiltrations (fig. 1). A computed tomography (CT) scan of the abdomen revealed the presence of a solid mass $4.9 \times 3.6 \mathrm{~cm}$ in size. The intravenous contrast agent administration enriched the masses which were located on the right side of the abdomen, behind the abdominal wall, in the mesentery adjacent to the intestinal helices but without affecting the lumen of the small intestine (fig. 2 ). These findings supported the suggestion that the tumors derived from the mesenchyme.

Due to intestinal involvement, a colonoscopy and gastroscopy was also performed, presenting normal findings. In order to establish a conclusive diagnosis, the surgeons proceeded to exploratory laparotomy through which the two masses were resected. The results of the histopathological analysis showed epithelioid granulomas, with or without caseous necrosis, with the presence of Langerhans-type multicore giant cells. These findings in association with the presence of fibrous elements in the chest X-ray prompted the suspicion of peritoneal TB. 
The patient was referred to the pulmonary department for further investigation. Tuberculin skin test was positive $(15 \mathrm{~mm})$ and a bronchoscopy was undertaken revealing normal findings. Ziehl-Neelsen staining and culture of the bronchial lavage were both negative for B-Koch. Thoracic CT scan showed pronounced calcified granulomas and fibrous elements in the right upper lobe (lesions of old TB), without presence of enlarged lymph nodes (fig. 3 ). The patient received fourfold antituberculous treatment with isoniazid, rifampicin, pyrazinamide and ethambutol and was discharged from the hospital to continue treatment at home for 6 months with instructions for regular follow-up.

\section{Discussion}

The diagnosis of any extrapulmonary forms of TB is especially difficult, particularly for the peritoneal type as the symptoms and physical findings of the disease do not substantiate diagnosis. Diagnostic delays and delayed initiation of treatment can lead to high morbidity and mortality rates [6]. The initial differential diagnosis of patients with nonspecific presentation of clinical symptoms such as vomiting, abdominal pain, ascites, weight loss and fever should also include peritoneal TB [7].

One or more of the following criteria can diagnose abdominal TB: (i) histological demonstration of tubercles with caseation necrosis, (ii) operative findings with biopsy of mesenteric nodes showing histologic evidence of TB, (iii) animal inoculation or culture of suspected tissue resulting in growth of M. tuberculosis, and (iv) histological evidence of acid-fast bacilli in a lesion. These criteria can be considered for the diagnosis, validated by radiologic and histopathological techniques [5].

CT findings of peritoneal TB include peritoneal thickening, ascites with fine septations, and omental caking [8]. Absence of radiographic findings does not exclude extrapulmonary TB as evidence of disease outside the abdomen is often not present and in $50 \%$ of the cases, chest X-ray is normal [1]. Ultrasonography is very useful for detecting peritoneal involvement. Barium studies generally do not detect tuberculous peritonitis and the tuberculin skin test is sensitive only in two thirds of cases [9]. M. tuberculosis on smear and culture of peritoneal fluid is usually insufficient and thus diagnostically low [10]. Adenosine deaminase activity in ascitic fluid has been recommended as a diagnostic test for peritoneal TB, however, according to the relevant literature, its usefulness remains controversial [11]. Polymerase chain reaction testing of the biopsy tissue and culture detects M. tuberculosis and is diagnostically useful in patients with ascites for a prompter diagnosis and treatment [12]. However, culture of the tubercles can sometimes be negative despite the presence of caseating granulomas [13]. Laparoscopic or laparotomic examination of the peritoneum is efficient to obtain a substantiated diagnosis. The finding of these examinations can be categorized in three groups: (i) studding peritoneum with multiple tubercles, (ii) thickened peritoneum, and (iii) fibroadhesive peritonitis [14]. Microbiological evidence and/or histological appearance of granulomas, with or without caseation, can determine the diagnosis [5].

The pharmaceutical treatment of abdominal TB recommends a conventional antituberculous therapy for at least 6 months including an initial 2 months of rifampicin, isoniazid, pyrazinamide and ethambutol. The duration of treatment may also be extended to 12 or 18 months [15]. Complications of abdominal TB include ulcer, perforation, adhesion, obstruction, bleeding, fistula formation and stenosis [5]. Surgical 
therapy is also necessary in some cases, especially for the treatment of disease complications such as perforation, obstruction, fistula or hemorrhage [1].

Peritoneal TB is a treatable infection, but its diagnosis is often delayed due to nonspecific biological markers, long incubation times for cultures and the absence of characteristic radiographic or ultrasonographic signs [15]. Therefore, the diagnosis may sometimes be postponed or even missed, significantly affecting the prognosis of the disease. In our case, the diagnosis of peritoneal TB was not suspected until operation. The absence of specific signs such as fever, swelling abdomen from ascites, anorexia and other intestinal symptoms prevented the surgeons from suspecting other diseases. Especially the absence of ascetic fluid accumulation, which is reported in more than $90 \%$ of cases, is a rare occasion and therefore, in future studies, should not rule out the differential diagnosis of abdominal TB. For this reason, specialized tests for the diagnosis of TB, e.g. tuberculin skin test or adenosine deaminase activity, were not initially undertaken.

Following the abdominal CT scan, an exploratory laparotomy was warranted as the technique allows full inspection of the peritoneal cavity and biopsies of the tubercles. Resection of the two masses was indicated as they were solid with clear and smooth boundaries without intestinal involvement. Previous relevant studies have reported that diagnosis is usually established at the time of surgery or that $25-75 \%$ of patients with abdominal TB eventually need surgery [2].

Peritoneal TB is a rare disease with no characteristic clinical features, and even after thorough investigation, the diagnosis can be elusive. A high index of suspicion is required to enable an accurate diagnosis. An effective therapy and increased survival rates depend exclusively on early diagnosis [6]. Most of the relevant diagnostic techniques can contribute valuable information, however, none of them alone can substantiate the diagnosis of peritoneal TB. Physicians must bear in mind that a combination of radiologic, endoscopic, microbiologic and histopathological examination achieves diagnostic accuracy and prevents clinical mismanagement.

\section{Acknowledgement}

The authors would like to thank their colleagues and nursing staff of the 1st Surgery Department, General University Hospital of Alexandroupolis, Greece.

\section{Disclosure Statement}

The authors have no conflicts of interest to declare. 


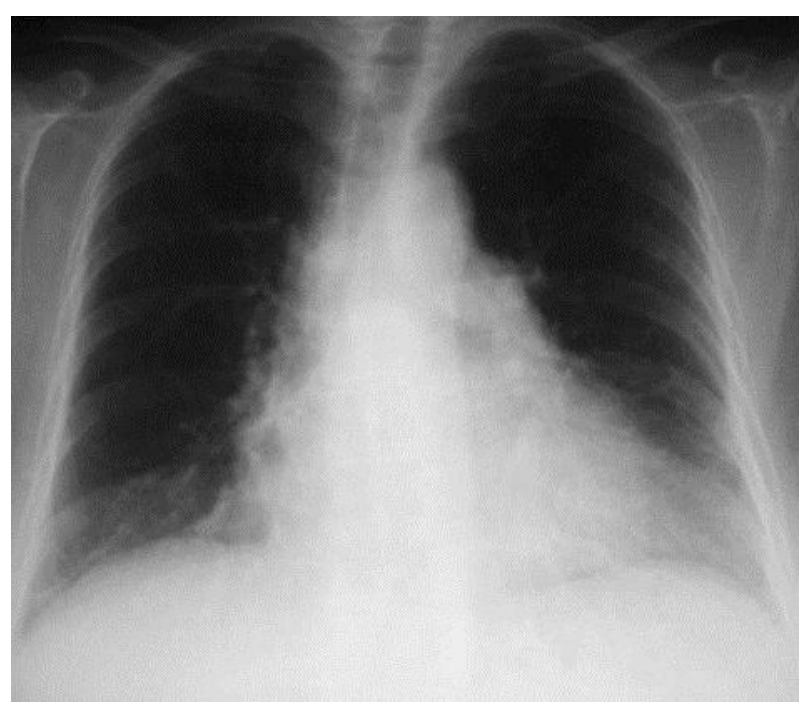

Fig. 1. Posterior-anterior chest X-ray not indicative of active pulmonary disease.

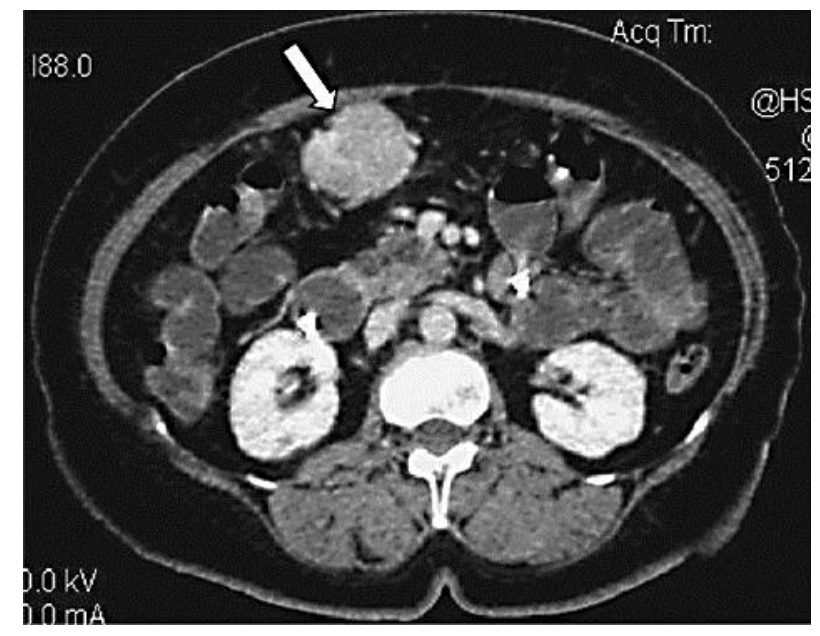

Fig. 2. Axial enhanced image of CT enteroclysis with neutral enteral contrast demonstrates a round soft tissue mass in the mesentery corresponding to a tuberculoma. The mass enhances inhomogeneously and does not involve small bowel loops. Areas of necrosis or calcifications are not seen. 


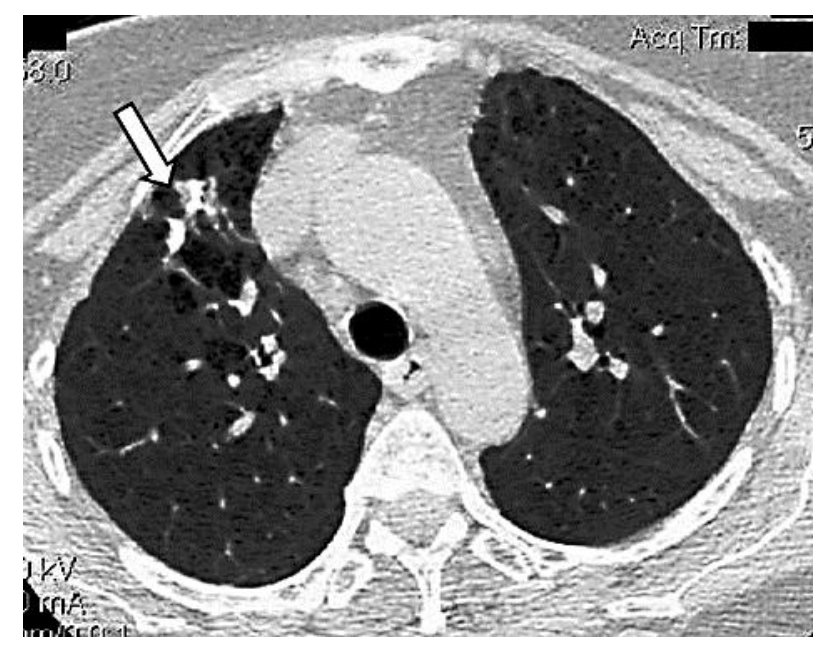

Fig. 3. Axial CT image of the chest shows calcifications and fibrosis (arrow) in the right upper lobe in a patient with history of TB.

\section{References}

1 Flores-Alvarez E, Tello-Brand SE, Lopez-Lopez F, Rivera-Barragan V: Peritoneal tuberculosis. Report of seven cases. Cir Cir 2010;78:67-71.

2 Farias Llamas OA, Lopez Ramirez MK, Morales Amezcua JM, Medina Quintana M, Buonocunto Vazquez G, Ruiz Chavez IE, Gonzalez Ojeda A: Peritoneal and intestinal tuberculosis: an ancestral disease that poses new challenges in the technological era. Case report and review of the literature (in Spanish). Rev Gastroenterol Mex 2005;70:169-179.

3 Bhatia MS, Srivastava S, Sharma S: 1. An uncommon case of zoophilia: a case report. Med Sci Law 2005;45:174-175.

4 Sharma MP, Bhatia V: Abdominal tuberculosis. Indian J Med Res 2004;120:305-315.

5 Sanai FM, Bzeizi KI: Systematic review: Tuberculous peritonitis - presenting features, diagnostic strategies and treatment. Aliment Pharmacol Ther 2005;22:685-700.

6 Rasheed S, Zinicola R, Watson D, Bajwa A, McDonald PJ: Intra-abdominal and gastrointestinal tuberculosis. Colorectal Dis 2007;9:773-783.

7 Khan R, Abid S, Jafri W, Abbas Z, Hameed K, Ahmad Z: Diagnostic dilemma of abdominal tuberculosis in non-HIV patients: an ongoing challenge for physicians. World J Gastroenterol 2006;12:6371-6375.

-8 Vazquez Munoz E, Gomez-Cerezo J, Atienza Saura M, Vazquez Rodriguez JJ: Computed tomography findings of peritoneal tuberculosis: systematic review of seven patients diagnosed in 6 years (19962001). Clin Imaging 2004;28:340-343.

-9 Malik A, Saxena NC: Ultrasound in abdominal tuberculosis. Abdom Imaging 2003;28:574-579.

10 Franco-Paredes C, Leonard M, Jurado R, Blumberg HM, Smith RM: Tuberculosis of the pancreas: report of two cases and review of the literature. Am J Med Sci 2002;323:54-58.

11 Vogel Y, Bous JC, Winnekendonk G, Henning BF: Tuberculous peritonitis in a German patient with primary biliary cirrhosis: a case report. J Med Case Rep 2008;2:32.

12 Uzunkoy A, Harma M: Diagnosis of abdominal tuberculosis: experience from 11 cases and review of the literature. World J Gastroenterol 2004;10:3647-3649.

13 Demir K, Okten A, Kaymakoglu S, Dincer D, Besisik F, Cevikbas U, Ozdil S, Bostas G, Mungan Z, Cakaloglu Y: Tuberculous peritonitis - reports of 26 cases, detailing diagnostic and therapeutic problems. Eur J Gastroenterol Hepatol 2001;13:581-585.

14 Bhargava DK, Shriniwas, Chopra P, Nijhawan S, Dasarathy S, Kushwaha AK: Peritoneal tuberculosis: laparoscopic patterns and its diagnostic accuracy. Am J Gastroenterol 1992;87:109-112.

15 Blumberg HM, Leonard MK Jr, Jasmer RM: Update on the treatment of tuberculosis and latent tuberculosis infection. JAMA 2005;293:2776-2784. 